WORKING PAPER $\cdot$ NO. 2020-53

Coordination and Social Distancing: Inertia in the Aggregate Response to COVID-19

Mehdi Shadmehr and Ethan Bueno de Mesquita

APRIL 2020

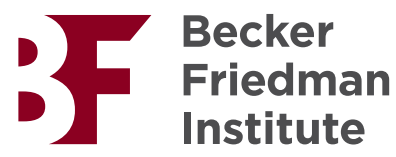




\title{
Coordination and Social Distancing: Inertia in the Aggregate Response to COVID-19*
}

\author{
Mehdi Shadmehr ${ }^{\dagger} \quad$ Ethan Bueno de Mesquita ${ }^{\ddagger}$
}

April 17, 2020

\begin{abstract}
Social distancing - which is critical for mitigating the spread of COVID-19 - has been slow and inadequate. Applying the literature on beauty contest models, we show: (1) When a new and rare virus, like COVID-19, emerges, the aggregate level of social distancing has inherent inertia; (2) Clear national public statements are essential in reducing that inertia and adjusting the public's behavior to the new, optimal level of social distancing; (3) National communication is better than local communication when optimal social distancing levels are highly correlated over-time and when individuals are poorly-informed.
\end{abstract}

*We have received helpful comments from Scott Ashworth, Dan Bernhardt, Wioletta Dziuda, Amanda Friedenberg, and Arda Gitmez. We thank the Becker-Friedman Institute for financial support.

${ }^{\dagger}$ Harris School of Public Policy, University of Chicago, and Department of Economics, University of Calgary. E-mail: mehdi@uchicago.edu

${ }^{\ddagger}$ Harris School of Public Policy, University of Chicago. Email:bdm@uchicago.edu 
Motivated by the COVID-19 outbreak, we study a model of social distancing in which people care about both engaging in the correct level of social distancing and conforming to the behavior of other people (i.e., adhering to social norms). When citizens care about these two considerations, and are uncertain about each others' beliefs about the right level of social distancing, aggregate social distancing exhibits significant inertia. In particular, following a large positive shock to the right level of social distancing (e.g., due to the outbreak of a contagious virus), aggregate social distancing will be far below the social optimum, even if individuals' information accurately reflects this shock on average (Proposition 3).

Citizens care not only about doing the right thing but also about conforming to social norms. For example, many feel awkward shopping in a mask, risking offending a neighbor by waiting for the next elevator, declining an outstretched hand, or forbidding playdates, if their fellow citizens are not going to do likewise. These concerns create inertia - citizens' actions overweight their common knowledge about the past social distancing behavior because doing so allows them to follow social norms by coordinating on similar levels of social distancing. This inertia is problematic because society would be better off coordinating on a new, higher, level of social distancing.

How can the government improve this situation? Our Proposition 4 suggests that public statements from prominent leaders (e.g., presidential speeches) about the new optimal level of social distancing reduce the inertia by generating new common knowledge that enables people to coordinate their actions around new, more appropriate norms.

Should such statements be made by national or local governments and leaders? That is, should the information be communicated more publicly or more privately? Proposition 5 suggests that national communication is better than local communication when optimal social distancing levels are highly correlated over-time and when individuals are poorlyinformed, so that the overweighting of prior social distancing norms is more severe. This result reflects that the information communicated publicly creates new common knowledge. As such, people concerned with conforming to social norms overweight the new public information. This overweighting can be beneficial because it helps offset the overweighting of prior social distancing norms.

Standard accounts frame the problem of social distancing as a public goods problem with the familiar externalities. For example, individuals do not internalize that social distancing has positive health spillovers on others, so there will be under-provision of social distancing. In this setting, the fundamental problem is not informational: even fullyinformed citizens under-provide social distancing absent some more heavy-handed policy that directly changes citizen incentives - e.g., forced downtown closure or fines for public gatherings. Moreover, enforcing behavioral changes for actions that are largely taken out of the public eye, such as hand washing, handshakes, or private gatherings, is virtually impossible. This aspect of the social distancing challenge has been the focus of public and academic discussions. For example, Allcott et al. (2020) study the interaction between risk perception and such externalities in the United States. Dube and Baicker (2020) discuss the importance of individuals sacrificing their interests for the greater good and, 
drawing on Christensen et al.'s (2020) study of the Ebola crisis in Sierra Leon, emphasize the importance of trust in local leaders and institutions.

In contrast, our analysis highlights the role of social norms and strategic uncertainty as an under-appreciated source of counter-productive inertia in aggregate social distancing. The policy implications are also sharply different. While information alone cannot resolve the standard externalities problem, clear and consistent public information can dramatically improve social distancing by reducing strategic uncertainty and enabling citizens to coordinate on new norms. Moreover, in contrast to accounts that emphasize the role of the local community in providing trusted information, we highlight the advantage of information provided by national over local leaders, especially in countries like United States where there is trust in the expertise of governmental health organizations. National coverage generates more common knowledge, enabling citizens to better coordinate on new optimal norms of social distancing.

What sorts of decisions might our norms-based model represent in the context of the COVID-19 crisis? The key features of the model are that: (1) people are uncertain about the right level of social distancing and are uncertain about what others believe, (2) the right level of social distancing exhibits serial correlation, and (3) there is a desire for conformity with social norms.

An important application of our model concerns social gatherings. The advent of COVID-19 made it undesirable, from a social welfare perspective, for people to participate in events such as St. Patrick's Day celebrations, spring break trips and parties, or sporting events. However, people were uncertain about the threat that COVID-19 posed. Crucially, to the extent that they believed others might think that the threat was modest and therefore continue to gather, they too had incentives to behave this way. The result was undesirable inertia - people continued past practices in ways that harmed social welfare.

Similar arguments hold for social practices such as hand shaking, kissing, and other forms of physical social greetings. It is awkward to refuse to shake an offered hand - as a recent presidential press briefing illustrated - creating a force for social conformity of the sort we model. The uncertainty about others' views on COVID-19 created unfortunate inertia in physical social greetings.

Our model also applies to some professional settings. If employees believe that continuing to come to the office signals commitment or ambition, then this creates social pressure for employees to continue coming to work if their managers or supervisors are. In the face of such pressure, the model suggests, there will be inertia that keeps people coming into the office at inefficiently high levels, especially during the early days of a viral outbreak. ${ }^{1}$

Finally, the model also helps understand inertia in social distancing and its policy implications, not only at the initial outset of a contagious virus, but at any point where

\footnotetext{
${ }^{1}$ In this setting, there could also be congestion externalities that we leave unmodeled - as fewer people start going into the office, the risk of being infected in the office goes down.
} 
there is a sharp change in the appropriate level of social distancing. As such, the policy implications will continue to apply in the event of intermittent future flare ups requiring renewed social distancing.

We utilize a standard "beauty contest" model in which individuals aim to take actions that match an uncertain state of the world (the right action) while remaining close to the average action in the population. Morris and Shin's (2002) seminal paper showed that individuals over-react to public information in such settings. Subsequent literature explored the welfare consequences of this insight and its implications for optimal communication (Morris and Shin 2007; Angeletos and Pavan 2007), as well as its extensions to dynamic settings (Morris and Shin 2006; Angeletos and La'O 2010; Huo and Pedroni 2020). This framework has been applied to study a wide range of topics from monetary policy (Lorenzoni 2010) to leadership in party conferences (Dewan and Myatt 2007), organizations (Bolton et al. 2013), and judiciaries (Shadmehr et al. 2019). Our paper provides an application in disease control and public health policy.

Our paper is also related to the game theoretic literature on social norms. Much of this literature interprets social norms as a set of equilibrium expectations and behaviors, so that different social norms correspond to different equilibria of a game with multiple equilibria (Myerson 1991, p. 113-114; Postlewaite 2011; Bidner and Franscois 2013; Acemoglu and Jackson 2015; Young 2015). We adopt Acemoglu and Jackson's (2017) definition of social norms as "the distribution of anticipated payoff-relevant behavior" (p. 246). Our model has a unique equilibrium, and social norms in our setting refer to the average (social distancing) behavior of the population. When agents are heterogeneously informed, the anticipated average behavior depends on the individual's information. We model people's desire to conform to social norms as a desire to do what is expected of them, as reflected in other people's behavior. The challenge of a global pandemic is that it creates uncertainty about what people expect of one another and how people will behave, leading people to lean on past, inappropriate, norms of behavior.

Acemoglu and Jackson (2015) study norms of cooperation in a dynamic setting where an agent in each period plays a complete information coordination game with agents of the immediate past and future periods. Their model features behavioral types who never cooperate and past actions are observed with noise, so some rational agents do not cooperate even in the best equilibrium. When the cooperative action of a generation becomes public for future generations, their expectations about cooperative behavior improves, facilitating cooperation - see also Acemoglu and Wolitsky (2014) and Acemoglu and Jackson (2017). In contrast to these papers, agents in our setting are uncertain about the right action and about one anothers' information in a changing world. While Acemoglu and Jackson (2015, 2017) focus on patterns of cooperation and compliance, our focus is on inertia and overreactions to public information in a world where optimal aggregate behavior evolves over time and on the resulting policy implications. 


\section{Model}

We apply the canonical framework and results discussed in Angeletos and Lian's (2016) comprehensive review of the literature. ${ }^{2}$ There is a continuum of citizens indexed by $i \in$ $[0,1]$, interacting over time, indexed by $t=0,1, \ldots$ In each period $t$, each citizen must take an action $a_{i t} \in \mathbb{R}$, which captures the degree of social distancing. A higher action corresponds to a higher level of social distancing. Absent concerns for conformity, the right action for each citizen is $\theta_{t}$. But, in each period, each citizen cares about targeting this right action and about conforming to the average action that others take in that period, $A_{t}=\int a_{i t} d i$. This generates a complementarity: if a citizen believes that others will do little social distancing, this raises that citizen's incentive also to do less social distancing. This captures, among other things, social pressure and the cost of deviating from the norms of behavior in the society. In particular, a citizen's payoff in period $t$ is:

$$
-(1-\alpha)\left(a_{i t}-\theta_{t}\right)^{2}-\alpha\left(a_{i t}-A_{t}\right)^{2},
$$

where $\alpha \in(0,1)$ is the citizens' relative weight on conformity.

The right action, $\theta_{t}$, follows a random walk: $\theta_{t}=\theta_{t-1}+u_{t}$, where $u_{t} \sim \operatorname{iidN}\left(0, \sigma_{u}\right)$. Citizens do not observe $\theta_{t}$, but each citizen observes a signal of the right action: $x_{i t}=\theta_{t}+\epsilon_{i t}$, where $\epsilon_{i t} \sim i i d N\left(0, \sigma_{\epsilon}\right)$. Throughout, we assume that the noise and fundamentals are independent from each other in the standard manner.

Citizen $i$ observes $x_{i t}$ in period $t$, and $\theta_{t-1}$ becomes public in period $t{ }^{3}$ Citizens discount future payoffs by $\delta$, and each citizen maximizes the expected sum of discounted period payoffs.

\section{Analysis}

We will think of the normative goal as each individual choosing the right level of aggregate social distancing, $a_{i t}=\theta_{t}$, so that the aggregate level of social distancing is also right, $A_{t}=\int a_{i t} d i=\theta_{t}$. This would be the objective of a policy maker who aggregates individual payoffs, but puts no weight on social conformity (i.e., sets $\alpha=0) .{ }^{4}$ In light of this, we say that any reduction in the expected quadratic distance between individual actions and the right action, $E\left[\int\left(a_{i t}-\theta_{t}\right)^{2} d i\right]$, is a normative improvement.

Our first result concerns the optimal level of social distancing under this normative criterion. Because the right action is uncertain, the normatively optimal individual actions - i.e.,

\footnotetext{
${ }^{2}$ This literature builds on the seminal work of Morris and Shin (2002).

${ }^{3}$ Even if $\theta_{t-1}$ is not observed in the current period, citizens will infer it in equilibrium if they observe the last period's aggregate behavior $A_{t-1}$.

${ }^{4}$ For our normative standard to coincide with the utilitarian social welfare, one can add a term $\alpha \int\left(a_{j t}-\right.$ $\left.A_{t}\right)^{2} d j$ to citizen payoffs in equation (1), so that citizens want the divergence of their own behavior from the average behavior to be close to the average divergence in the population.
} 
the actions that minimize $E\left[\int\left(a_{i t}-\theta_{t}\right)^{2} d i\right]$-involve citizens choosing their best estimate of the right action, $a_{i t}=E\left[\theta_{t} \mid x_{i t}, \theta_{t-1}\right]=\beta x_{i t}+(1-\beta) \theta_{t-1}$, where $\beta=\frac{\sigma_{u}^{2}}{\sigma_{u}^{2}+\sigma_{\epsilon}^{2}}$.

Proposition 1 (Normative Benchmark) If citizens did not care about conformity $(\alpha=$ $0)$, the aggregate action would be: $A_{t}=\theta_{t}+\beta u_{t}$, where $\beta=\frac{\sigma_{u}^{2}}{\sigma_{u}^{2}+\sigma_{\epsilon}^{2}}$.

We now analyze how citizens actually behave, given their concern for taking the right action and for social conformity. Because there is a continuum of citizens, a citizen's action does not affect the aggregate outcome, either in the current or in future periods. Thus, the only link between periods is information. From equation (1), citizen $i$ chooses the following degree of social distancing:

$$
a_{i t}=(1-\alpha) \mathbb{E}_{i t}\left[\theta_{t}\right]+\alpha \mathbb{E}_{i t}\left[A_{t}\right]
$$

where $\mathbb{E}_{i t}[\cdot]$ is the expectation of $i$ in period $t$ given his information.

Define $\overline{\mathbb{E}}^{h}$ recursively as follows. $\overline{\mathbb{E}}^{0}[X]=X, \overline{\mathbb{E}}^{1}[X]=\overline{\mathbb{E}}\left[\overline{\mathbb{E}}^{0}[X]\right]=\int \mathbb{E}_{i}[X] d i, \overline{\mathbb{E}}^{h}[X]=$ $\overline{\mathbb{E}}\left[\overline{\mathbb{E}}^{h-1}[X]\right]=\int \mathbb{E}_{i}\left[\overline{\mathbb{E}}^{h-1}[X]\right] d i$. That is, $\overline{\mathbb{E}}^{1}[X]$ is the average expectation of the random variable $X$ in the population; $\overline{\mathbb{E}}^{2}[X]$ is the average expectation in the population about the average expectation in the population, and so on. Proposition 2 shows that the aggregate social distancing in the population depends on all such higher order expectations in the population. The proof comes from iterating on equation (2). (All proofs are in the appendix.)

Proposition 2 (Higher Order Beliefs in Aggregate Action) The aggregate action in each period depends on all average higher order beliefs in the population about the right action, with lower weights on higher orders:

$$
A_{t}=\sum_{h=1}^{\infty}(1-\alpha) \alpha^{h-1} \overline{\mathbb{E}}_{t}^{h}\left[\theta_{t}\right]
$$

Observe that

$$
\mathbb{E}_{i t}\left[\theta_{t}\right]=\mathbb{E}_{i t}\left[u_{t}\right]+\theta_{t-1} \Rightarrow \overline{\mathbb{E}}_{t}^{h}\left[\theta_{t}\right]=\overline{\mathbb{E}}_{t}^{h}\left[u_{t}\right]+\theta_{t-1} .
$$

Now, using properties of Normal distribution and Proposition 2 yields:

Proposition 3 (Aggregate Actions Exhibit Excess Inertia) Conformity generates inertia. In particular,

- $A_{t}=\theta_{t-1}+\phi u_{t}$, where $0<\phi<\beta<1, \phi=\frac{(1-\alpha) \beta}{1-\alpha \beta}$, and $\beta=\frac{\sigma_{u}^{2}}{\sigma_{u}^{2}+\sigma_{\epsilon}^{2}}$.

- $\phi$ is decreasing in $\alpha$, with $\lim _{\alpha \rightarrow 0} \phi(\alpha)=\beta$ and $\lim _{\alpha \rightarrow 1} \phi(\alpha)=0$. 
Because citizens care about coordinating their actions, they put extra weight on their common knowledge of the right action in the past, which facilitates coordination - reminiscent of the logic of focal points. Citizens have common knowledge that, on average, the right action today is the right action yesterday (i.e., $\theta_{t} \sim N\left(\theta_{t-1}, \sigma_{u}\right)$ ) and hence over-weight this fact. As a result, today's aggregate action is biased in the direction of yesterday's right action. As a consequence, following a large positive shock, like COVID-19, to the right amount of social distancing, the aggregate action will be lower than the right action. ${ }^{5}$

Now, suppose in each period $t$, in addition to the their private signals $x_{i t}$, citizens also receive a public signal $p_{t}=\theta_{t}+\eta_{t}$, with $\eta_{t} \sim \operatorname{iidN}\left(0, \sigma_{\eta}\right)$. Such a public signal might be the result of information conveyed by the government. Proposition 2 and equation (4) still hold because they do not depend on the details of available information. However, the presence of public signals changes the degree of inertia in aggregate social distancing.

\section{Proposition 4 (Public Announcements)}

1. Public signals reduce inertia. Averaging over the public signal noise, the expected aggregate social distancing is: $E\left[A_{t} \mid \theta_{t-1}, u_{t}\right]=\theta_{t-1}+\phi_{p} u_{t}$, where $\phi_{p}>\phi$.

2. The amount of inertia is decreasing in the clarity of the public signal. That is, $\phi_{p}$ is monotone decreasing in $\sigma_{\eta}, \lim _{\sigma_{\eta} \rightarrow \infty} \phi_{p}\left(\sigma_{\eta}\right)=\phi$, and $\lim _{\sigma_{\eta} \rightarrow 0} \phi_{p}\left(\sigma_{\eta}\right)=1$.

3. Improving the clarity of the public signal causes a normative improvement (i.e., $E\left[\int\left(a_{i t}-\theta_{t}\right)^{2} d i\right]$ is increasing in $\left.\sigma_{\eta}\right)$ if: (i) $\alpha \leq 1 / 2$ or (ii) $\sigma_{\eta}$ is sufficiently small.

Proposition 4 shows that, following a shock, an informed leader can send a public signal that helps set public expectations about the aggregate right action, thereby reducing inertia in social distancing driven by the desire to conform. The clearer that message (i.e., the lower $\sigma_{\eta}$ ), the more this will reduce inertia.

Such public messages are a normative improvement if people don't put too much weight on conformity $(\alpha \leq 1 / 2)$ or the public signal is sufficiently informative $\left(\sigma_{\eta}\right.$ small). Why these conditions? Because citizens value conformity, they put excessive weight on all public signals relative to a Bayesian individual who only cares about choosing an action that reflects the best estimate of $\theta_{t}$ (this was the same logic that drove inertia in the first place). Because this distortion is smaller when $\alpha$ is smaller, new public information about the optimal social distancing is always beneficial when citizens put relatively less weight on conformity $(\alpha \leq 1 / 2)$. In the other extreme, when citizens almost only care about conformity $(\alpha \approx 1)$, they put almost no weight on their private signals. Now, although citizens over-weight new public information $\left(p_{t}\right)$, this over-reaction to the new public information helps counter-act their over-reaction to past experience (that $\theta_{t} \sim\left(\theta_{t-1}, \sigma_{u}^{2}\right)$ ), and the overall effect is again beneficial. In between, when $\alpha \in(1 / 2,1)$, these effects compete and the

\footnotetext{
${ }^{5}$ The over-weighting of public information is a key insight of Morris and Shin (2002) who showed that public announcements can be damaging to welfare, particularly, in financial settings.
} 
overall effect of raising the precision of new public information may be negative unless it is sufficiently informative ( $\sigma_{\eta}$ small) to offset the over-reaction. ${ }^{6}$

For social distancing in the presence of a dangerous infectious disease, we believe the relevant parameter space is $\alpha \leq 1 / 2$. It is unlikely that people care so much about conformity that over-reaction to new public information trumps its value. Hence, for cases like COVID-19, Proposition 4 suggests that clear and consistent public messages from a leader are socially beneficial.

Given the overreaction by citizens to public messages described above, one may wonder whether there is a better way for the government to deliver information. Would it be better for citizens to receive the same level of information, but privately rather than publicly? For instance, perhaps employers or local governments could provide private information to citizens, rather than them all observing the same public information in a presidential speech or press conference.

To consider this possibility, contrast the public signal case with a setting where, instead of receiving private and public signals $x_{i t} \sim N\left(\theta_{t}, \sigma_{\epsilon}^{2}\right)$ and $p_{t} \sim N\left(\theta_{t}, \sigma_{\eta}^{2}\right)$, citizens receive a single private signal $x_{i t}^{\prime}$ with the same amount of information about the right action $\theta_{t}$ as the public and private signals combined. In particular, let $x_{i t}^{\prime}=\theta_{t}+\epsilon_{i t}^{\prime}$, with $\epsilon_{i t}^{\prime} \sim$ $N\left(0, \sigma_{\epsilon^{\prime}}^{2}=\frac{\sigma_{\epsilon}^{2} \sigma_{\eta}^{2}}{\sigma_{\epsilon}^{2}+\sigma_{\eta}^{2}}\right)$.

Proposition 5 The setting with the combination of private and public signals $\left(x_{i t}, p_{t}\right)$ is a normative improvement over the setting with more precise private signals $x_{i t}^{\prime}$ when $\sigma_{u}$ is sufficiently small or $\sigma_{\epsilon}$ is sufficiently large.

When citizens believe the past is highly informative about the present $\left(\sigma_{u}\right.$ small) or that they are privately poorly-informed $\left(\sigma_{\epsilon}\right.$ large), citizens put too much weight on their past experience. In such circumstances, it is better for the government to communicate publicly rather than privately. Citizens over-react to the government's public messages. But that will help to counter-act their over-reaction to their past experience. By contrast, when citizens believe the past is relatively uninformative $\left(\sigma_{u}\right.$ large) or that they are privately well-informed ( $\sigma_{\epsilon}$ small), the government should communicate privately.

In the context of social distancing in the wake of a new and rare infectious disease, individuals' information is typically very noisy ( $\sigma_{\epsilon}$ is large) and the appropriate level of social distancing is very sticky ( $\sigma_{u}$ is low, the disease is a very unusual shock). As such, for social distancing in the wake of COVID-19, clear and consistent public statements by a national leader are more effective than statements by local governments or employers, not because the national government is more informed, but because clear national statements generate over-reaction that is beneficial to correct the inertia created by the over-weighting of past habits and social norms.

\footnotetext{
${ }^{6}$ Equation (16) in the proof of Proposition 4 shows the necessary and sufficient conditions for when reducing $\sigma_{\eta}$ is a normative improvement.
} 


\section{References}

Acemoglu, Daron, and Matthew O. Jackson. 2015. "History, Expectations, and Leadership in the Evolution of Social Norms." Review of Economic Studies 82: 1-34.

Acemoglu, Daron, and Matthew O. Jackson. 2017. "Social Norms and the Enforcement of Laws." Journal of the European Economic Association 12: 245-95.

Acemoglu, Daron, and Alexander Wolitzky. 2014. "Cycles of Conflict: An Economic Model." American Economic Review 104: 1350-67.

Allcott, Hunt, Levi Boxell, Jacob Conway, Matthew Gentzkow, Michael Thaler, and David Yang. 2020. "Polarization and Public Health: Partisan Differences in Social Distancing during the Coronavirus Pandemic." Mimeo.

Angeletos, George-Marios, and Alessandro Pavan. 2007. "Efficient Use of Information and Social Value of Information." Econometrica 75: 1103-42.

Angeletos, George-Marios, and Jennifer La'O. 2010. "Noisy Business Cycles." in NBER Macroeconomics Annual 2009, Volume 24, pp. 319-378, University of Chicago Press.

Angeletos, George-Marios, and Chen Lian. 2016. "Incomplete Information in Macroeconomics: Accommodating Frictions in Coordination." Handbook of Macroeconomics, Vol. 2: $1065-1240$.

Bidner, Chris, and Patrick Francois. 2013. "The Emergence of Political Accountability." Quarterly Journal of Economics 128: 1397-1448.

Bolton, Patrick, Markus K. Brunnermeier, and Laura Veldkamp. 2013. "Leadership, Coordination and Corporate Culture." Review of Economic Studies 80: 512-37.

Christensen, Darin, Oeindrila Dube, Johannes Haushofer, Bilal Siddiqi, and Maarten Voors. 2020. "Building Resilient Health Systems: Experimental Evidence from Sierra Leone and the 2014 Ebola Outbreak." Mimeo.

Dewan, Torun, and David P. Myatt. 2007. "Leading the Party: Coordination, Direction, and Communication." American Political Science Review 101: 827-45.

Dube, Oeindrila, and Katherine Baicker. 2020. "How You Can Protect Your Community, Not Just Your Own Health." New York Times, March 26, 2020.

Huo, Zhen, and Marcelo Pedroni. 2020. "A Single-Judge Solution to Beauty Contests." American Economic Review 110: 526-68.

Lorenzoni, Guido. 2010. "Optimal Monetary Policy with Uncertain Fundamentals and Dispersed Information. Review of Economic Studies 77: 305-38.

Morris, Stephen, and Hyun Song Shin. 2002. "The Social Value of Public Information." American Economic Review 92: 1521-34. 
Morris, Stephen, and Hyun Song Shin. 2006. "Inertia of Forward-Looking Expectations." AEA Papers \& Proceedings 96: 152-7.

Morris, Stephen, and Hyun Song Shin. 2007. "Optimal Communication." Journal of the European Economic Association 5: 594-602.

Myerson, Roger. 1991. Game Theory: Analysis of Conflict. Boston, MA: Harvard University Press.

Postlewaite, Andrew. 2011. "Social Norms and Preferences." Handbook of Social Economics, Volume 1A, edited by J. Benhabib, A. Bisin, and M. Jackson. Amsterdam: NorthHolland.

Shadmehr, Mehdi, Charles Cameron, and Sepehr Shahshahani. 2019. "Coordination and Innovation in Judiciaries: Correct Law vs. Consistent Law." Mimeo.

Young, Peyton. 2015. "The Evolution of Social Norms." Annual Review of Economics 7: 359-87.

\section{Appendix: Proofs}

Proof of Proposition 2: From equation (2),

$$
A_{t}=\int a_{i t} d i=\int\left((1-\alpha) \mathbb{E}_{i t}\left[\theta_{t}\right]+\alpha \mathbb{E}_{i t}\left[A_{t}\right]\right) d i=(1-\alpha) \overline{\mathbb{E}}_{t}\left[\theta_{t}\right]+\alpha \overline{\mathbb{E}}_{t}\left[A_{t}\right]
$$

Iterating yields:

$$
A_{t}=(1-\alpha) \overline{\mathbb{E}}_{t}\left[\theta_{t}\right]+(1-\alpha) \alpha \overline{\mathbb{E}}_{t}^{2}\left[\theta_{t}\right]+\alpha^{2} \overline{\mathbb{E}}_{t}^{2}\left[A_{t}\right]
$$

Repeated iteration yields the result.

Proof of Proposition 3: We calculate $\overline{\mathbb{E}}_{t}^{h}\left[\theta_{t}\right]$, and use Proposition 2. Note that $x_{i t}=$ $\theta_{t}+\epsilon_{i t}=\theta_{t-1}+u_{t}+\epsilon_{i t}$. Thus, letting $\beta=\frac{\sigma_{u}^{2}}{\sigma_{u}^{2}+\sigma_{\epsilon}^{2}}$

$$
\mathbb{E}_{i t}\left[u_{t}\right]=\beta\left(x_{i t}-\theta_{t-1}\right)=\beta\left(u_{t}+\epsilon_{i t}\right) \Rightarrow \overline{\mathbb{E}}_{t}\left[u_{t}\right]=\beta u_{t}
$$

Iterating yields:

$$
\overline{\mathbb{E}}_{t}^{h}\left[u_{t}\right]=\beta^{h} u_{t}
$$

Substituting from equation (5) into equation (4) yields:

$$
\overline{\mathbb{E}}_{t}^{h}\left[\theta_{t}\right]=\beta^{h} u_{t}+\theta_{t-1}
$$

Now, substituting from equation (6) into equation (3) in Proposition 2 yields:

$$
A_{t}=\sum_{h=1}^{\infty}(1-\alpha) \alpha^{h-1}\left(\beta^{h} u_{t}+\theta_{t-1}\right)=\theta_{t-1}+\frac{\beta(1-\alpha)}{1-\alpha \beta} u_{t}
$$


In equation (7), let $\phi=\frac{(1-\alpha) \beta}{1-\alpha \beta}$, and observe that $\lim _{\sigma_{\epsilon} \rightarrow 0} \beta=\lim _{\sigma_{u} \rightarrow \infty} \beta=1$.

Proof of Proposition 4: Part 1. With the public signal $p_{t}, \mathbb{E}_{i t}\left[u_{t}\right]=E\left[u_{t} \mid x_{i t}, p_{t}\right]$. Thus,

$$
\begin{aligned}
\mathbb{E}_{i t}\left[u_{t}\right] & =\frac{\sigma_{u}^{2} \sigma_{\eta}^{2}\left(x_{i t}-\theta_{t-1}\right)+\sigma_{u}^{2} \sigma_{\epsilon}^{2}\left(p_{t}-\theta_{t-1}\right)}{\sigma_{u}^{2} \sigma_{\eta}^{2}+\sigma_{u}^{2} \sigma_{\epsilon}^{2}+\sigma_{\epsilon}^{2} \sigma_{\eta}^{2}} \\
& =\frac{\sigma_{u}^{2} \sigma_{\eta}^{2}\left(u_{t}+\epsilon_{i t}\right)+\sigma_{u}^{2} \sigma_{\epsilon}^{2}\left(p_{t}-\theta_{t-1}\right)}{\sigma_{u}^{2} \sigma_{\eta}^{2}+\sigma_{u}^{2} \sigma_{\epsilon}^{2}+\sigma_{\epsilon}^{2} \sigma_{\eta}^{2}} .
\end{aligned}
$$

Thus,

$$
\overline{\mathbb{E}}_{t}\left[u_{t}\right]=\frac{\sigma_{u}^{2} \sigma_{\eta}^{2} u_{t}+\sigma_{u}^{2} \sigma_{\epsilon}^{2}\left(p_{t}-\theta_{t-1}\right)}{\sigma_{u}^{2} \sigma_{\eta}^{2}+\sigma_{u}^{2} \sigma_{\epsilon}^{2}+\sigma_{\epsilon}^{2} \sigma_{\eta}^{2}}=A_{u} u_{t}+A_{p}\left(p_{t}-\theta_{t-1}\right),
$$

where

$$
A_{u}=\frac{\sigma_{u}^{2} \sigma_{\eta}^{2}}{\sigma_{u}^{2} \sigma_{\eta}^{2}+\sigma_{u}^{2} \sigma_{\epsilon}^{2}+\sigma_{\epsilon}^{2} \sigma_{\eta}^{2}} \text { and } A_{p}=\frac{\sigma_{u}^{2} \sigma_{\epsilon}^{2}}{\sigma_{u}^{2} \sigma_{\eta}^{2}+\sigma_{u}^{2} \sigma_{\epsilon}^{2}+\sigma_{\epsilon}^{2} \sigma_{\eta}^{2}}
$$

with

$$
\lim _{\sigma_{\eta} \rightarrow \infty} A_{p}=0, \lim _{\sigma_{\eta} \rightarrow \infty} A_{u}=\beta, \lim _{\sigma_{\eta} \rightarrow 0} A_{p}=1, \text { and } \lim _{\sigma_{\eta} \rightarrow 0} A_{u}=0 .
$$

Iterating on equation (9) yields

$$
\overline{\mathbb{E}}_{t}^{h}\left[u_{t}\right]=\left(A_{u}\right)^{h} u_{t}+\left(1+\cdots+A_{u}^{h-1}\right) A_{p}\left(p_{t}-\theta_{t-1}\right)
$$

Substituting from equation (12) into equation (4) yields:

$$
\begin{aligned}
\overline{\mathbb{E}}_{t}^{h}\left[\theta_{t}\right] & =\left(A_{u}\right)^{h} u_{t}+\left(1+\cdots+A_{u}^{h-1}\right) A_{p}\left(p_{t}-\theta_{t-1}\right)+\theta_{t-1} \\
& =\left(A_{u}\right)^{h} u_{t}+\frac{1-A_{u}^{h}}{1-A_{u}} A_{p}\left(p_{t}-\theta_{t-1}\right)+\theta_{t-1} .
\end{aligned}
$$

Now, substituting from equation (13) into equation (3) in Proposition 2 yields:

$$
\begin{aligned}
A_{t} & =\sum_{h=1}^{\infty}(1-\alpha) \alpha^{h-1}\left(\left(A_{u}\right)^{h} u_{t}+\frac{1-A_{u}^{h}}{1-A_{u}} A_{p}\left(p_{t}-\theta_{t-1}\right)+\theta_{t-1}\right) \\
& =\theta_{t-1}+\frac{(1-\alpha) A_{u}}{1-\alpha A_{u}} u_{t}+(1-\alpha) \frac{A_{p}}{1-A_{u}}\left(\frac{1}{1-\alpha}-\frac{A_{u}}{1-\alpha A_{u}}\right)\left(p_{t}-\theta_{t-1}\right) \\
& =\theta_{t-1}+\frac{(1-\alpha) A_{u}}{1-\alpha A_{u}} u_{t}+\frac{A_{p}}{1-\alpha A_{u}}\left(p_{t}-\theta_{t-1}\right) \\
& =\theta_{t-1}+\frac{(1-\alpha) A_{u} u_{t}+A_{p}\left(p_{t}-\theta_{t-1}\right)}{1-\alpha A_{u}}
\end{aligned}
$$

Note that, using (11), if $\sigma_{\eta} \rightarrow \infty$, equation (14) simplifies to equation (7).

For given $\theta_{t-1}$ and $u_{t}$, aggregate action $A_{t}$ takes different values for different values of the public signal $p_{t}$, depending on the idiosyncratic error term $\eta_{t}$ in the public signal. The 
average public signal, for given $\theta_{t-1}$ and $u_{t}$, is $E\left[p_{t} \mid u_{t}\right]=\theta_{t-1}+u_{t}$. Then, averaging over the public signal noise, equation (14) becomes:

$$
E\left[A_{t} \mid u_{t}, \theta_{t-1}\right]=\theta_{t-1}+\phi_{p} u_{t}, \text { where } \phi_{p}=\frac{(1-\alpha) A_{u}+A_{p}}{1-\alpha A_{u}} .
$$

Part 2. From (11), $\lim _{\sigma_{\eta} \rightarrow 0} \phi_{p}=1$ and $\lim _{\sigma_{\eta} \rightarrow \infty} \phi_{p}=\phi$. Comparing with $\phi$ in Proposition 3 yields:

$$
\phi_{p}-\phi=\frac{(1-\alpha) A_{u}+A_{p}}{1-\alpha A_{u}}-\frac{(1-\alpha) \beta}{1-\alpha \beta}=\frac{\sigma_{\epsilon}^{4} \sigma_{u}^{2}}{\left(\sigma_{\epsilon}^{2}+(1-\alpha) \sigma_{u}^{2}\right)\left(\sigma_{\epsilon}^{2} \sigma_{u}^{2}+\sigma_{\eta}^{2}\left(\sigma_{\epsilon}^{2}+(1-\alpha) \sigma_{u}^{2}\right)\right)}>0 .
$$

That is, $\phi<\phi_{p}$. Moreover,

$$
\frac{d \phi_{p}}{d \sigma_{\eta}^{2}}=-\frac{\sigma_{\epsilon}^{4} \sigma_{u}^{2}}{\left.\sigma_{\epsilon}^{2} \sigma_{u}^{2}+\sigma_{\eta}^{2}\left(\sigma_{\epsilon}^{2}+(1-\alpha) \sigma_{u}^{2}\right)\right)^{2}}<0
$$

Thus, reducing the noise in the public signal (less $\left.\sigma_{\eta}^{2}\right)$ raises $\phi_{p}$.

Part 3. From (2),

$$
\begin{aligned}
a_{i t} & =(1-\alpha) \mathbb{E}_{i t}\left[\theta_{t}\right]+\alpha \mathbb{E}_{i t}\left[A_{t}\right] \\
& =(1-\alpha) \mathbb{E}_{i t}\left[\theta_{t-1}+u_{t}\right]+\alpha \mathbb{E}_{i t}\left[\theta_{t-1}+\frac{(1-\alpha) A_{u} u_{t}+A_{p}\left(p_{t}-\theta_{t-1}\right)}{1-\alpha A_{u}}\right] \quad \text { (from (14)) } \\
& =\theta_{t-1}+(1-\alpha) \mathbb{E}_{i t}\left[u_{t}\right]+\alpha \frac{(1-\alpha) A_{u}}{1-\alpha A_{u}} \mathbb{E}_{i t}\left[u_{t}\right]+\alpha \frac{A_{p}\left(p_{t}-\theta_{t-1}\right)}{1-\alpha A_{u}} \\
& =\theta_{t-1}+\frac{1-\alpha}{1-\alpha A_{u}} \mathbb{E}_{i t}\left[u_{t}\right]+\alpha \frac{A_{p}\left(p_{t}-\theta_{t-1}\right)}{1-\alpha A_{u}} \\
& =\theta_{t-1}+\frac{1-\alpha}{1-\alpha A_{u}}\left(A_{u}\left(u_{t}+\epsilon_{i t}\right)+A_{p}\left(p_{t}-\theta_{t-1}\right)\right)+\alpha \frac{A_{p}}{1-\alpha A_{u}}\left(p_{t}-\theta_{t-1}\right) \quad \text { (from (8) and (10)) } \\
& =\theta_{t-1}+\frac{(1-\alpha) A_{u}}{1-\alpha A_{u}} u_{t}+\frac{(1-\alpha) A_{u}}{1-\alpha A_{u}} \epsilon_{i t}+\frac{A_{p}}{1-\alpha A_{u}}\left(p_{t}-\theta_{t-1}\right) .
\end{aligned}
$$

Thus,

$$
\begin{aligned}
a_{i t}-\theta_{t} & =\frac{(1-\alpha) A_{u}}{1-\alpha A_{u}} u_{t}+\frac{(1-\alpha) A_{u}}{1-\alpha A_{u}} \epsilon_{i t}+\frac{A_{p}}{1-\alpha A_{u}}\left(p_{t}-\theta_{t-1}\right)-u_{t} \\
& \left.=\frac{\left(A_{p}+A_{u}-1\right) u_{t}+A_{p} \eta_{t}+(1-\alpha) A_{u} \epsilon_{i t}}{1-\alpha A_{u}} \text { (substituting } p_{t}-\theta_{t-1}=u_{t}+\eta_{t}\right) .
\end{aligned}
$$

Thus,

$$
\begin{aligned}
\left(a_{i t}-\theta_{t}\right)^{2} & =\frac{\left(A_{p}+A_{u}-1\right)^{2} u_{t}^{2}+A_{p}^{2} \eta_{t}^{2}+(1-\alpha)^{2} A_{u}^{2} \epsilon_{i t}^{2}}{\left(1-\alpha A_{u}\right)^{2}} \\
& +\frac{2\left(A_{p}+A_{u}-1\right) u_{t} A_{p} \eta_{t}+2\left(A_{p}+A_{u}-1\right) u_{t}(1-\alpha) A_{u} \epsilon_{i t}+2 A_{p} \eta_{t}(1-\alpha) A_{u} \epsilon_{i t}}{\left(1-\alpha A_{u}\right)^{2}} .
\end{aligned}
$$


Thus,

$$
\int\left(a_{i t}-\theta_{t}\right)^{2} d i=\frac{\left(A_{p}+A_{u}-1\right)^{2} u_{t}^{2}+A_{p}^{2} \eta_{t}^{2}+(1-\alpha)^{2} A_{u}^{2} \sigma_{\epsilon}^{2}+2\left(A_{p}+A_{u}-1\right) A_{p} u_{t} \eta_{t}}{\left(1-\alpha A_{u}\right)^{2}} .
$$

Thus,

$$
E\left[\int\left(a_{i t}-\theta_{t}\right)^{2} d i\right]=\frac{\left(A_{p}+A_{u}-1\right)^{2} \sigma_{u}^{2}+A_{p}^{2} \sigma_{\eta}^{2}+(1-\alpha)^{2} A_{u}^{2} \sigma_{\epsilon}^{2}}{\left(1-\alpha A_{u}\right)^{2}},
$$

where we recognize that if $\alpha=0$, equation (15) simplified to $\frac{\sigma_{u}^{2} \sigma_{\eta}^{2} \sigma_{\epsilon}^{2}}{\sigma_{u}^{2} \sigma_{\eta}^{2}+\sigma_{u}^{2} \sigma_{\epsilon}^{2}+\sigma_{\epsilon}^{2} \sigma_{\eta}^{2}}$, which is the variance of $\theta_{t} \mid \theta_{t-1}, p_{t}, x_{i t}$. Differentiating with respect to $\sigma_{\eta}^{2}$ yields:

$$
\frac{d E\left[\int\left(a_{i t}-\theta_{t}\right)^{2} d i\right]}{d \sigma_{\eta}^{2}}=\frac{\sigma_{\epsilon}^{4} \sigma_{u}^{4}\left(\sigma_{\epsilon}^{2} \sigma_{u}^{2}+\sigma_{\epsilon}^{2} \sigma_{\eta}^{2}+(1-\alpha)(1-2 \alpha) \sigma_{u}^{2} \sigma_{\eta}^{2}\right)}{\left(\sigma_{\epsilon}^{2} \sigma_{u}^{2}+\sigma_{\eta}^{2} \sigma_{\epsilon}^{2}+(1-\alpha) \sigma_{\eta}^{2} \sigma_{u}^{2}\right)^{3}} .
$$

Thus, if $\sigma_{\epsilon}^{2}+(1-\alpha)(1-2 \alpha) \sigma_{u}^{2} \geq 0$ (in particular, if $\alpha \leq 1 / 2$ ), the above derivative is strictly positive. If, instead, $\sigma_{\epsilon}^{2}+(1-\alpha)(1-2 \alpha) \sigma_{u}^{2}<0$, the above derivative is strictly positive if and only if $\sigma_{\eta}^{2}$ is sufficiently small.

Proof of Proposition 5: To obtain $E\left[\int\left(a_{i t}-\theta_{t}\right)^{2} d i\right]$ with only $x_{i t}^{\prime}$, first let $\sigma_{\eta} \rightarrow \infty$ in (15), and then substitute $\sigma_{\epsilon}^{2}$ with $\sigma_{\epsilon^{\prime}}^{2}=\frac{\sigma_{\epsilon}^{2} \sigma_{\eta}^{2}}{\sigma_{\epsilon}^{2}+\sigma_{\eta}^{2}}$. Using (11), and recognizing that $\lim _{\sigma_{\eta} \rightarrow \infty} A_{p} \sigma_{\eta}^{2}=$ 0 , the first step yields:

$$
\lim _{\sigma_{\eta} \rightarrow \infty} \frac{(\beta-1)^{2} \sigma_{u}^{2}+(1-\alpha)^{2} \beta^{2} \sigma_{\epsilon}^{2}}{(1-\alpha \beta)^{2}}=\frac{\sigma_{\epsilon}^{2} \sigma_{u}^{2}\left(\sigma_{\epsilon}^{2}+(1-\alpha)^{2} \sigma_{u}^{2}\right)}{\left(\sigma_{\epsilon}^{2}+(1-\alpha) \sigma_{u}^{2}\right)^{2}} .
$$

Substituting $\sigma_{\epsilon}^{2}$ with $\sigma_{\epsilon^{\prime}}^{2}$ yields:

$$
\frac{\sigma_{\epsilon^{\prime}}^{2} \sigma_{u}^{2}\left(\sigma_{\epsilon^{\prime}}^{2}+(1-\alpha)^{2} \sigma_{u}^{2}\right)}{\left(\sigma_{\epsilon^{\prime}}^{2}+(1-\alpha) \sigma_{u}^{2}\right)^{2}}
$$

Now, subtracting (15) from (17) yields:

$$
\begin{aligned}
& \Delta=E\left[\int\left(a_{i t}-\theta_{t}\right)^{2} d i\right]_{\left(x_{i t}^{\prime}\right)}-E\left[\int\left(a_{i t}-\theta_{t}\right)^{2} d i\right]_{\left(x_{i t}, p_{t}\right)} \\
= & \frac{\alpha^{2} \sigma_{\eta}^{4} \sigma_{\epsilon}^{4} \sigma_{u}^{4}}{\left(\sigma_{\eta}^{2} \sigma_{\epsilon}^{2}+(1-\alpha)\left(\sigma_{\eta}^{2}+\sigma_{\epsilon}^{2}\right) \sigma_{u}^{2}\right)^{2}\left(\sigma_{\epsilon}^{2} \sigma_{u}^{2}+\sigma_{\eta}^{2}\left(\sigma_{\epsilon}^{2}+(1-\alpha) \sigma_{u}^{2}\right)\right)^{2}}\left(\sigma_{\epsilon}^{4}\left(\sigma_{\eta}^{2}+\sigma_{u}^{2}\right)-(1-\alpha)^{2}\left(\sigma_{\eta}^{2}+\sigma_{\epsilon}^{2}\right) \sigma_{u}^{4}\right) .
\end{aligned}
$$

As expected, $\lim _{\alpha \rightarrow 0} \Delta=0$, because only the amount information matter; and $\lim _{\alpha \rightarrow 1} \Delta>$ 0 , because then citizens put a lot of weight of the pre-existing public information from the previous period, which need to be countered by new public information about $\theta_{t}$. Moreover, for any $\alpha>0$, the setting with both public and private signals $\left(x_{i t}, p_{t}\right)$ is a normative improvement over the setting with only private signals $\left(x_{i t}^{\prime}\right)$ if and only if $\Delta>0$, i.e., if and only if

$$
\left(\frac{\sigma_{\epsilon}^{2}}{\sigma_{u}^{2}}\right)^{2}>(1-\alpha)^{2} \frac{\sigma_{\eta}^{2}+\sigma_{\epsilon}^{2}}{\sigma_{\eta}^{2}+\sigma_{u}^{2}}
$$

The result follows from inspection of this inequality. 